\title{
EXTENSIONS OF HIAI-LIN TYPE EIGENVALUE INEQUALITY
}

\section{JIAN SHI}

Abstract. In this paper, we prove several extensions of Hiai-Lin type eigenvalue inequality which extends the relative result before.

Mathematics subject classification (2010): 47A63.

Keywords and phrases: Eigenvalues, Tanahashi inequality, Hiai-Lin type inequality.

\section{REFERENCES}

[1] F. HiAI, M. Lin, On an eigenvalue inequality involving the Hadamard product, Linear Algebra Appl. 515 (2017), 313-320.

[2] K. Tanahashi, The Furuta inequality with negative powers, Proc. Amer. Math. Soc. 127 (1999), $1683-1692$. 